\title{
The Assessment of Intrapartum Transperineal Ultrasonographic Parameters for their Effectiveness in Evaluation of Progress of Labor and Prediction of Mode of Delivery in Egyptian Women
}

Gamal Abdelsameea Ibrahim, Ahmed Soliman Nasr, Fatma Atta*, Mohamed Reda (D), Hend Abdelghany, Nihal M. El-Demiry, Mohamed Shalaby

Department of Obstetrics and Gynecology, Kasr el Ainy Faculty of Medicine, Cairo University, Giza, Egypt

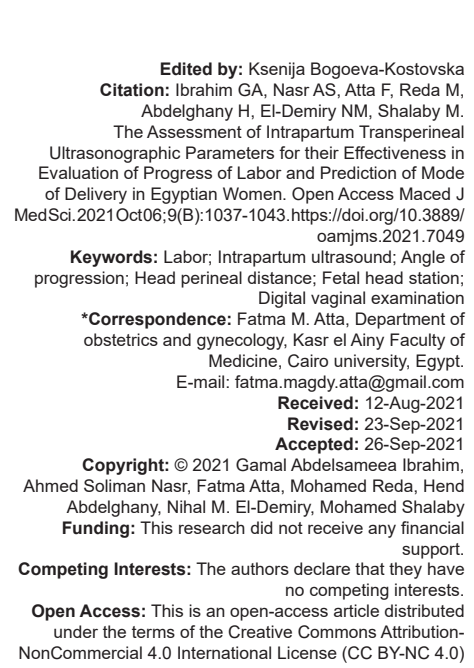

Introduction

During normal labor, regular and efficient uterine contractions cause progressive dilation and effacement of the cervix, accompanied by descent and eventual expulsion of the fetus. "Abnormal labor," "dystocia," and "failure to progress" are terms that have been traditionally used to describe labor patterns deviating from that observed in the majority of women who have a spontaneous vaginal delivery. These labor abnormalities are best described as protraction disorders (i.e., slower than normal progress) or arrest disorders (i.e., complete cessation of progress) [1].

\begin{abstract}
(FHS) has been associated with prolonged labor and delivery outcomes. Although clinical assessment of FHS is both subjective and unreliable, women with prolonged labor are subjected to multiple digital vaginal examinations (dVEs). The use of ultrasound has been proposed to aid in the management of labor since 1990s. Ultrasound examination is more accurate and reproducible than clinical examination in the those women destined for spontaneous vaginal delivery and those destined for operative delivery and may predict the outcome of instrumental vaginal delivery. Such a technique has the potential to reduce the frequency of intrusive internal examinations and associated infection and could be useful in allowing the assessment of women in whom dVE is traumatic or contra-indicated. Intrapartum ultrasound not only provides objective and quantitative data in and fetus as a supplementary tool for active management.
\end{abstract}

AIM: This study aims at assessing the value of intrapartum transperineal ultrasonography as a quantitative and

PATIENTS: This study was a prospective observational study conducted on 600 primiparous women in active first or admitted to Kasr Al Ainy maternity hospital from January 2017 to June 2018. The studied population was divided into two groups: Group A of 300 women with normal progress of labor and Group B of 300 women with

METHODS: FHS was assessed clinically by dVE and sonographically by transperineal ultrasound measurement of head perineal distance (HPD) and angle of progression (AOP). Intrapartum care of the patient continued as norma based only on dVEs using the modified WHO partogram. Statistical analysis was targeted toward assessing the potential of the intrapartum ultrasonography in the evaluation of progress of labor and prediction of mode of delivery.

RESULTS: All studied parameters for assessment of FHS (dVE, HPD, and AOP) significantly correlated with each other and with both progress of labor and mode of delivery with $p(<0.001)$. The highest sensitivity for prediction of progress of labor is observed using dVE (83\%) and the highest specificity is observed using AOP (78.3\%). The ( parameters was found to have a high sensitivity of $97.7 \%$ and a high positive predictive value of $86.63 \%$.

CONCLUSION: Intrapartum ultrasound examination is a valuable tool in the prediction of progress of labor and mode of delivery. The assessment of FHS by transperineal ultrasound measurement of HPD and AOP is much more informative of the progress of labor and the mode of delivery than digital assessment of FHS.
Although women who experience labor dystocia may ultimately deliver vaginally, a longer first stage of labor is associated with adverse maternal and neonatal outcomes [1]. When progress failure or fetal distress occurs, obstetricians should choose between an operative vaginal delivery (OVD) or a cesarean section (CS). Slow progress of labor is one of the leading causes of CS [2].

Although it was in 1976 that Friedman and Sachtleben described an association between high fetal head station (FHS) in prolonged labor and delivery outcome in nulliparous women, there is still no objective method of predicting the likelihood of vaginal 
delivery. Clinical assessment of FHS by digital vaginal examination (dVE) is both subjective and unreliable [3] Despite this, women with prolonged labor are subjected to multiple dVEs, which often serve little purpose other than to document a dysfunctional labor.

The use of ultrasound has been proposed to aid in the management of labor since 1990s. Several studies have demonstrated that ultrasound examination is more accurate and reproducible than clinical examination in the diagnosis of fetal head position and station and in the prediction of arrest of labor [4]. Ultrasound examination can, to some extent, distinguish those women destined for spontaneous vaginal delivery and those destined for operative delivery. Furthermore, there is growing evidence that ultrasound in labor may predict the outcome of instrumental vaginal delivery [5]. Such a technique has the potential to reduce the frequency of intrusive internal examinations and associated infection and could be useful in allowing the assessment of women in whom dVE is traumatic or contra-indicated [3]. Despite these advantages, in the delivery room, ultrasound support still plays a secondary role with respect to clinical evaluation [6]. With increasing resolution and easier accessibility, ultrasound is receiving more and more attention. Intrapartum ultrasound not only provides objective and quantitative data in labor, but also helps to make more reliable clinical decisions aiming to improve obstetric outcomes of both the mother and fetus as a supplementary tool for active management.

\section{Aim of the work}

This study aims at assessing the value of intrapartum transperineal ultrasonography as a quantitative and objective tool in the evaluation of progress of labor and prediction of mode of delivery.

\section{Subjects and Methods}

\section{Subjects}

This study was a prospective observational case-control study conducted on 600 primiparous women in active first stage of labor admitted to Kasr Al Ainy maternity hospital from January 2017 to June 2018 after the approval of the ethical committee. Included subjects were primiparous women between 18 and 35 years of age with term singleton pregnancy of a living fetus with cephalic presentation. Excluded from the study were women having risk factors for labor dystocia as fetal macrosomia, malpresentation, oligohydramnios and suspected cephalopelvic disproportion or any absolute indication for CS. The 600 women were assigned to two groups according to progress of labor where women with normal progress of labor were assigned to group $A$ and those were 300 while those with prolonged $1^{\text {st }}$ stage of labor were assigned to Group B and were also 300.

\section{Methods}

After obtaining a full informed consent, all women enrolled in the study were subjected to Full history taking for fulfillment of the inclusion and exclusion criteria. Full general, and abdominal examination was performed together with dVE for assessment of cervical position, dilatation, consistency and length, condition of membranes, pelvic adequacy and presentation, position and detection of FHS. Transperineal ultrasonographic parameters were measured in the active first stage of labor, in between contractions, and just before or after applying the dVE. Transperineal ultrasonography was performed by a single operator for all patients enrolled in the study by using a 5-9 Hz convex abdominal probe and a Samsung SonoAce R3 portable ultrasound machine. Recommended ultrasound settings were lowest possible angle of insonation, lowest output frequency, highest insonation depth, and wide volumetric area with low sound volume. In our study, we assessed the head perineum distance (HPD) and angle of progression (AOP). HPD is the shortest distance between the outer bony limit of the fetal skull and the perineum. AOP is the angle between a line through the midline of the pubic symphysis and a line from the inferior apex of the symphysis to the leading part of the fetal skull. The mean of three measurements from separate acquisitions was determined and recorded. The health professionals responsible for the care of the patient were blinded to the ultrasound findings and continued to provide care as normal based only on dVEs using the modified WHO partogram [7] for assessment of labor progress. The investigators, on the other hand, were uninvolved in the women management and decisions taken by the caring physicians. After data collection, our statistical analysis was targeted towards assessing the potential of the intrapartum ultrasonography as a quantitative and objective tool in the evaluation of progress of labor and prediction of mode of delivery.

\section{Results}

Our study population was divided into two groups (Group A and B) according to progress of labor, and demographic, maternal, fetal, labor, and delivery characteristics were recorded. Table 1 shows these characteristics regarding the whole study population, across the two groups and according to mode of delivery.

Demonstrated in Table 2 is the study population regarding the mode of delivery where 458 women had a 
Table 1: Maternal, fetal, and labor characteristics of the whole population and across groups and according to mode of delivery

\begin{tabular}{|c|c|c|c|c|c|c|}
\hline \multirow[t]{2}{*}{ Characteristics } & \multirow[t]{2}{*}{ Whole population } & \multicolumn{2}{|l|}{ Progress of labor } & \multicolumn{3}{|l|}{ Mode of delivery } \\
\hline & & Group A & Group B & VD & CS & OVD \\
\hline Maternal age (years) & $24.08 \pm 4.41$ & $23.64 \pm 4.46$ & $24.51 \pm 4.32$ & $23.62 \pm 4.17$ & $25.87 \pm 4.61$ & $24.40 \pm 5.56$ \\
\hline Maternal body mass index & $28.76 \pm 3.38$ & $27.82 \pm 2.87$ & $29.69 \pm 3.59$ & $28.33 \pm 3.20$ & $30.29 \pm 3.54$ & $29.63 \pm 3.74$ \\
\hline Gestational age (weeks) & $38.74 \pm 1.28$ & $38.48 \pm 1.14$ & $38.99 \pm 1.37$ & $38.62 \pm 1.24$ & $39.04 \pm 1.32$ & $39.30 \pm 1.44$ \\
\hline Estimated fetal weight (grams) & $3203.56 \pm 268.93$ & $3139.25 \pm 220.98$ & $3267.87 \pm 296.24$ & $3169.61 \pm 239.36$ & $3345.35 \pm 329.83$ & 3192 \\
\hline AFI & $7.53 \pm 1.87$ & $7.55 \pm 1.79$ & $7.50 \pm 1.94$ & $7.58 \pm 1.75$ & $7.25 \pm 2.33$ & \pm 280 \\
\hline Duration of active $1^{\text {st }}$ stage $(\mathrm{h})$ & 10.6 & 8.94 & 12.77 & 10.22 & 15.56 & $7.70 \pm 1.70$ \\
\hline Duration of $2^{\text {nd }}$ stage $(\mathrm{h})$ & 1.37 & 0.94 & 1.95 & 1.28 & 1.94 & 12 \\
\hline \multicolumn{7}{|l|}{ Initiation of labor } \\
\hline Spont. & $68 \%$ & $76 \%$ & $59.7 \%$ & $72.9 \%$ & $49.1 \%$ & $60 \%$ \\
\hline Induced & $32 \%$ & $24 \%$ & $40.3 \%$ & $27.1 \%$ & $50.9 \%$ & $40 \%$ \\
\hline \multicolumn{7}{|l|}{ Augment. of labor } \\
\hline Yes & $57 \%$ & $18.7 \%$ & $95.3 \%$ & $48.3 \%$ & $92 \%$ & $60 \%$ \\
\hline No & $43 \%$ & $81.3 \%$ & $4.7 \%$ & $51.7 \%$ & $8 \%$ & $40 \%$ \\
\hline
\end{tabular}

Table 2: Mode of delivery of the whole population and across groups

\begin{tabular}{lllll}
\hline Groups & \multicolumn{2}{l}{ Mode of delivery } & p value \\
\cline { 2 - 4 } & VD & CS & OVD & \\
\hline Whole population & $76 \%$ & $19 \%$ & $5 \%$ & \\
Progress of labor & & & & \\
Group A & $93 \%$ & $2.7 \%$ & $4.3 \%$ & $<0.001$ \\
$\quad$ Group B & $59.7 \%$ & $34.7 \%$ & $5.7 \%$ & \\
\hline OVD: Operative vaginal delivery, CS: Cesarean section. & & &
\end{tabular}

vaginal delivery $(76.3 \%)$, whereas 30 had an operative vaginal delivery (5\%), and 112 had a CS (18.7\%). Indications for delivery by CS or OVD are demonstrated in Figure 1.

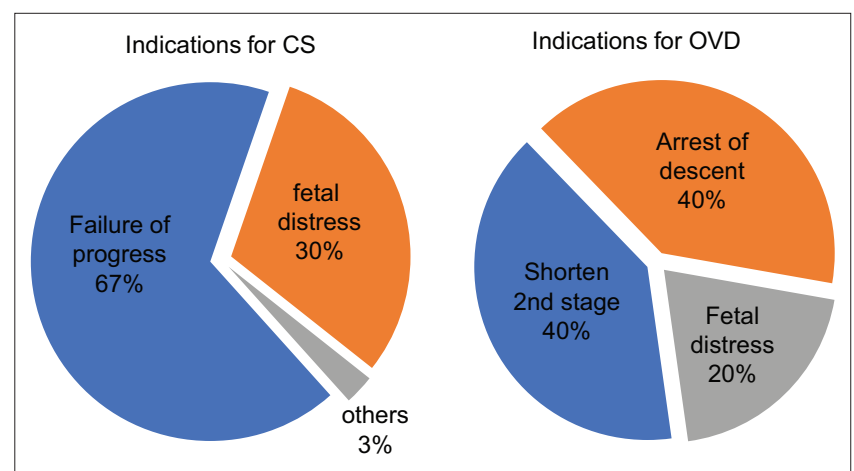

Figure 1: Indications for cesarean section and operative vaginal delivery

In our study, the evaluation of FHS was done using clinical and ultrasonographic parameters. Clinical assessment of FHS was done by dVE while the ultrasonographic parameters used were measurement of HPD and AOP. Table 3 demonstrates the clinical and ultrasonographic parameters in the whole study population, across groups, and according to mode of delivery.

The value of clinical and ultrasonographic parameters in prediction of Progress of Labor and mode of delivery was noted and demonstrated in Table 4. While the highest sensitivity for prediction of progress of labor is observed using dVE (83\%), the highest specificity is observed using AOP (78.3\%). The highest sensitivity for prediction mode of delivery is for combined HPD and AOP (97.7\%) while the highest specificity is for AOP (81\%) (Figure 2).

When combining both HPD and AOP for prediction of vaginal delivery, the assessment of both parameters was found to have a high sensitivity of
$97.7 \%$ and a high positive predictive value of $86.63 \%$ (Figure 3).

\section{Discussion}

Traditionally, the assessment and management of a woman in labor are based on clinical findings. However, clinical examination of head station is inaccurate and subjective with uncertain reproducibility [5].

Although there were numerous studies conducted in this area of research, currently, there is no consensus regarding when in labor ultrasound should be performed, which parameter(s) should be obtained, and how the sonographic findings should be integrated into clinical practice to improve management of the patient [5].

ISUOG Practice Guidelines of intrapartum ultrasound was published in March 2018 and recommended that indications for ultrasound evaluation in labor include slow progress or arrest of labor in the first stage, slow progress or arrest of labor in the second stage, ascertainment of fetal head position and station before considering or performing instrumental vaginal delivery, and objective assessment of fetal head malpresentation [5].

Our study represented the initial experience of the use of intrapartum ultrasonography in Cairo University Hospitals. In our study, we tried to approach the dark spots in order to answers the above mentioned questions in addition to establishing the baseline characteristics in the Egyptian population.

Regarding the basic maternal, fetal, and labor characteristics, our cohort fairly resembled cohorts of similar studies regarding maternal body mass index, fetal gestational age, and estimated fetal weight. However, in our cohort, maternal age was much less than maternal age in similar studies. This significant difference may be attributed to the social background difference between different populations but does not appear to significantly affect the study outcome.

Regarding the outcome of labor, our study rates of spontaneous vaginal delivery showed similarity 
Table 3: Clinical and ultrasonographic characteristics of the whole population, across groups and mode of delivery

\begin{tabular}{|c|c|c|c|c|c|c|c|c|}
\hline \multirow[t]{2}{*}{ Characteristics } & \multirow[t]{2}{*}{ Whole population } & \multicolumn{3}{|c|}{ Progress of labor } & \multicolumn{4}{|c|}{ Mode of delivery } \\
\hline & & Group A & Group B & $p$ & VD & CS & OVD & $\mathrm{p}$ \\
\hline Clinical (Station 0) & $49 \%$ & $55.3 \%$ & $42.7 \%$ & $<0.001$ & $50.2 \%$ & $38.4 \%$ & $70 \%$ & $<0.001$ \\
\hline \multicolumn{9}{|l|}{ Ultrasound } \\
\hline $\begin{array}{l}\text { Head perineum distance } \\
\text { Angle of progression }\end{array}$ & $\begin{array}{l}3.9 \pm 0.88 \\
107.83 \pm 12.95\end{array}$ & $\begin{array}{l}3.48 \pm 0.78 \\
114.65 \pm 12.89\end{array}$ & $\begin{array}{l}4.32 \pm 0.76 \\
101.01 \pm 8.74\end{array}$ & $\begin{array}{l}<0.001 \\
<0.001\end{array}$ & $\begin{array}{l}3.8 \pm 0.87 \\
110.14 \pm 12.96\end{array}$ & $\begin{array}{l}4.39 \pm 0.79 \\
98.47 \pm 8.82\end{array}$ & $\begin{array}{l}3.61 \pm 0.47 \\
107.53 \pm 9.83\end{array}$ & $\begin{array}{l}<0.001 \\
<0.001\end{array}$ \\
\hline
\end{tabular}

Table 4: Clinical and ultrasonographic parameters for prediction of mode of delivery

\begin{tabular}{|c|c|c|c|c|c|c|}
\hline \multirow[t]{2}{*}{ Parameter } & \multicolumn{3}{|c|}{ Progress of labor } & \multicolumn{3}{|c|}{ Mode of delivery } \\
\hline & Cut-off & Sensitivity \% & Specificity \% & Cutoff & Sensitivity \% & Specificity \% \\
\hline Station & -0.5 & 83.5 & 52.2 & -0.5 & 71.5 & 52.8 \\
\hline Head perineum distance & 3.81 & 77 & 70.7 & 3.95 & 62.2 & 63.4 \\
\hline Angle of progression & 106.5 & 70.3 & 78.3 & 107.5 & 52.1 & 81 \\
\hline Combined head perineum distance and angle of progression & & 74.3 & 76.3 & & 97.7 & 51.4 \\
\hline
\end{tabular}

to those described in the similar studies; however, the operative vaginal delivery rates were much less with a higher CS rate to compensate for the low OVD rate. Out of the 600 laboring women, only 30 patients (5\%) had an OVD. This is a very low rate when compared to $17.3 \%$ reported by Youssef et al. [8], 33\% reported by Eggebø et al. [9], and $42.5 \%$ reported by Tutschek et al. [10]. This might be attributed to low availability of adequately trained personnel on OVD together with limited resources.

The CS rate in our study was low (18.7\%) compared to the CS rates according to the statistics of the labor ward in our department (approximately 40\%) and the published national statistics which approximates $52 \%$ [11]. This is attributed to the properties of the selected cohort in our study in which all of the absolute and most of the relative indications of CS were excluded from the study. However, when comparing this CS rate with matching cohorts in other studies it is still significantly higher. The CS rate in a similar cohort was found to be $7.7 \%$ in a study performed by Youssef et al. [8] and 18\% in the study of Gizzo et al. [6]. However, it was higher in the study of Eggebø et al. [9] where it was $26 \%$ because the study only included subjects with prolonged labor and this percentage is still lower when compared to the corresponding group in our study (Group B had CS rate of $37.4 \%$ ).

Normal progress of labor does not necessarily mean that the mode of delivery is vaginal and vice versa. This can be explained by the presence of other factors that might influence the mode of delivery other than the progress of labor such as the occurrence of fetal distress or the need to shorten the second stage despite normally progressing. Thus, the evaluation of the intrapartum ultrasonographic parameters by comparing groups regarding both the progress of labor and mode of delivery is more descriptive and multidimensional.

Regarding the assessment of FHS by transperineal ultrasonographic measurement of HPD and AOP, the measurement of HPD and AOP was successfully obtained in all of our subjects. This was similarly observed by Eggebø et al. [9].

Regarding the agreement between the ultrasonographic parameters and the dVE, our data analysis revealed statistically significant agreement between both HPD and AOP and FHS assessed by $\mathrm{dVE}$. These results were similarly observed by Hjartardóttir et al. [4]. Both HPD and AOP correlated significantly with the FHS. It was noticed that as the FHS increases, there was a decrease in the HPD and increase in AOP. Statistical analysis was done to investigate this correlation to reach cutoff values of these parameters that may reflect or even substitute the subjective assessment of FHS by VE. Of particular practical interest is the FHS 0 which indicates fetal head engagement. Our analysis showed that for station 0 , the mean HPD was $3.76 \pm 0.66 \mathrm{~cm}$, and the mean AOP was $108.14^{\circ} \pm 12.19^{\circ}$. These results were similarly observed by Tutschek et al. [10] where FHS 0 was corresponding to HPD $3.6 \mathrm{~cm}$ and AOP $116^{\circ}$. Similarly, a moderate correlation was observed between both HPD and AOP and dVE in the systematic review and meta-analysis conducted by Wiafe et al. [12]. Tutschek et al., 2013, found a good correlation between the ultrasound parameters measuring the degree of fetal head descent, but the ultrasound methods correlated only moderately with clinical assessments of FHS [10].

We observed a clear relationship between HPD and AOP with normal progress of labor. In labors that were progressing normally, HPD was found lower and $A O P$ was found wider than their corresponding measurements in prolonged labors. It was also clear that when the HPD is less and the AOP is wide, the labor is more likely to proceed in a normal pattern.

In our study, we observed a clear relationship between HPD and AOP with vaginal delivery. When the HPD was less and the AOP was wide, spontaneous vaginal delivery was more likely to occur. Inversely, when HPD was greater and AOP was narrower, CS was more likely to be the mode of delivery. Our statistical analysis showed that a cutoff value of HPD of $3.95 \mathrm{~cm}$ has sensitivity of $62 \%$, specificity of $63 \%$, and PPV of $84.5 \%$ in prediction of mode of delivery. Furthermore, our statistical analysis showed that a cutoff value of AOP of $107.5^{\circ} \mathrm{cm}$ has sensitivity of $52 \%$, specificity of $81 \%$, and PPV of $89.8 \%$ in prediction of mode of delivery. These results are in agreement with 


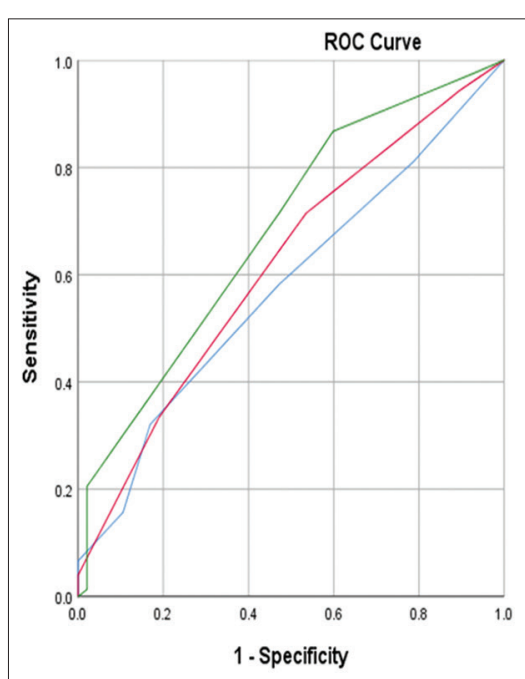

a

Diagonal segments are produced by ties

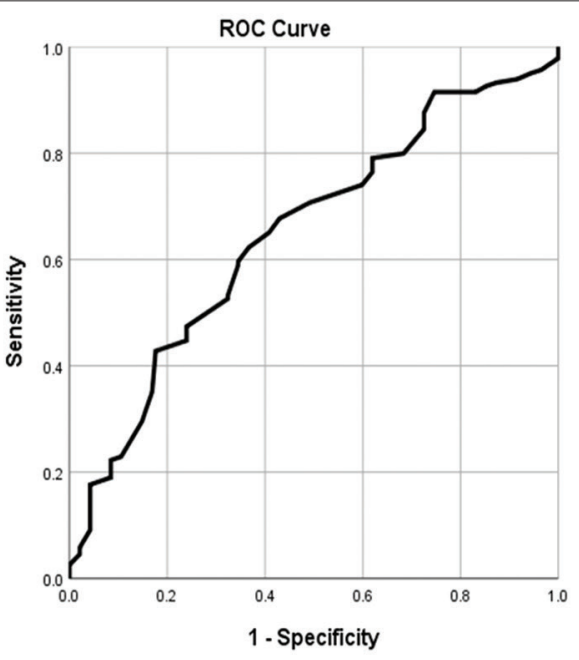

b Diagonal segments are produced by ties.

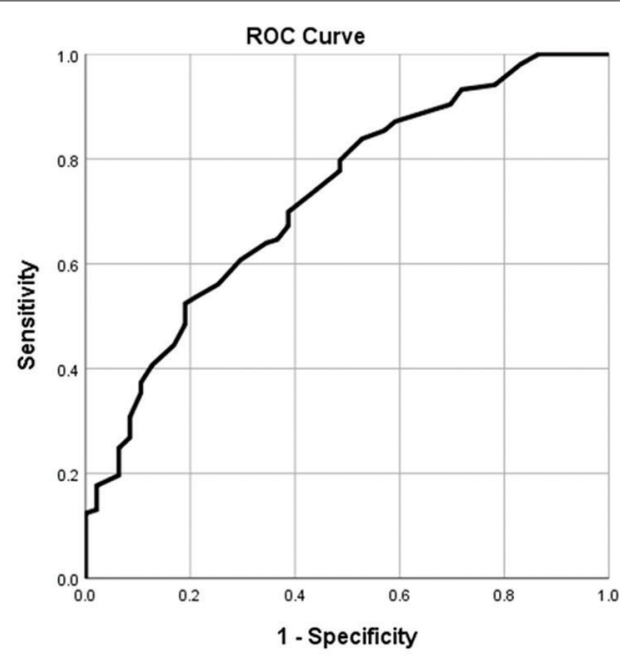

C

Diagonal segments are produced by ties.

Figure 2: (a-c) Receiver operating characteristic curve for prediction of mode of delivery using clinical parameters, head perineum distance, and angle of progression, respectively

the results of Eggebø et al. where HPD $\leq 4 \mathrm{~cm}$ was found to have a sensitivity of $69 \%$ and a PPV of $92 \%$ in prediction of vaginal delivery. Similarly, AOP of $\geq 110^{\circ}$ was found to have sensitivity of $68 \%$ and a PPV of $88 \%$ in prediction of vaginal delivery. Thus, Eggebø et al. found that AOP $>110^{\circ}$ and HPD $<40 \mathrm{~mm}$ were both good predictors of vaginal delivery in prolonged first stage of labor [9].

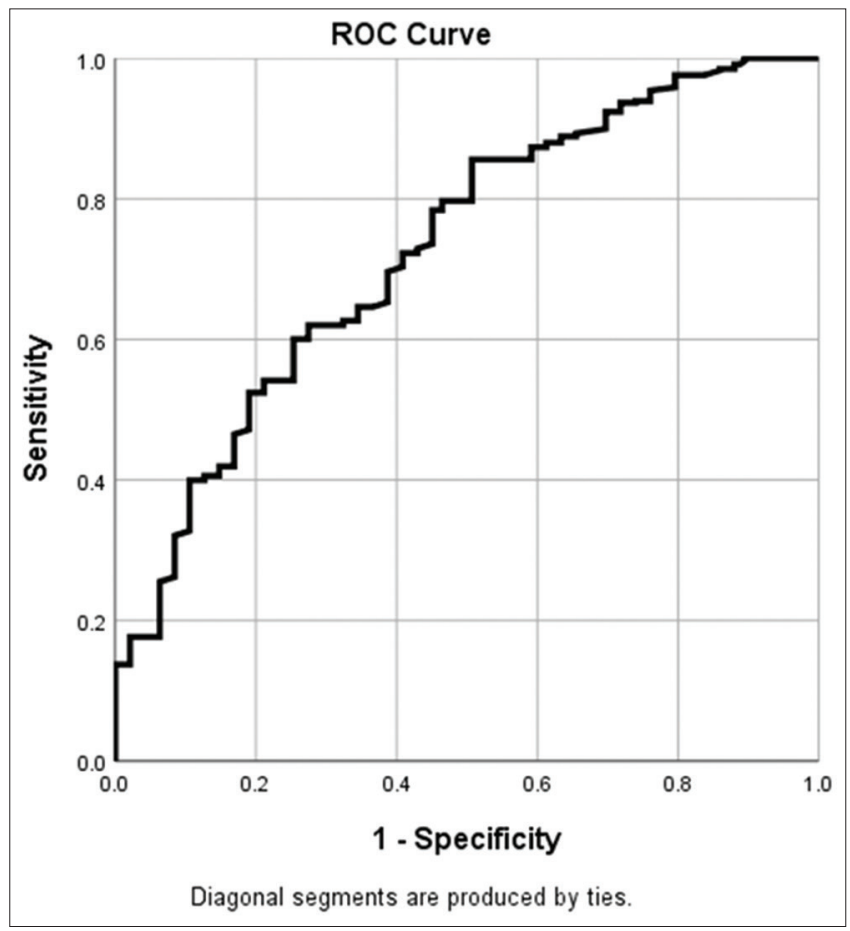

Figure 3: Receiver operating characteristic curve for prediction of mode of delivery using combined head perineum distance and angle of progression

Compared to measuring each parameter alone, combining HPD and AOP increased their predictive potential of vaginal delivery. Combined HPD and AOP were found to have sensitivity of $97.7 \%$ and PPV reached $86.63 \%$ for prediction of mode of delivery.

\section{Conclusion}

Intrapartum ultrasound examination is a valuable tool in the prediction of progress of labor and mode of delivery. The assessment of FHS by transperineal ultrasound measurement of HPD and AOP is much more informative of the progress of labor and the mode of delivery than digital assessment of FHS. This may be of particular importance regarding decision making and active interventions especially in situations where there is a suspected delay or arrest of first or second stage or the potential need for performance of OVD. Integration of ultrosonography within the clinical partogram is of recognized feasibility, reproducibility and availability.

\section{Recommendations}

We recommend the implementation of the intrapartum ultrasonography practice guide lines into the routine clinical practice in the management of labor and in the objective evaluation of the laboring women together with proper documentation of the parameters. The knowledge and training on intrapartum ultrasound technique and interpretation should be integrated in the basic postgraduate training programs in our department. Further research should be conducted taking in consideration the time to delivery, and the perinatal outcome. 


\section{Strengths, Weaknesses, and Limitations of the Study}

\section{Strengths of the study}

$\bullet$

The intrapartum ultrasound was done by a single operator thus avoiding interobserver bias. While, the health professionals responsible for the care of were blinded to the ultrasound findings. Therefore, their decisions were not influenced by our findings. The research team, on the other hand, was totally uninvolved in the decision and only recorded the labor outcome. The subjects continued to receive care as normal from the attending health professional and the management of labor was based only on the dVEs and clinical data collected routinely Large sample size with two groups which allowed for the more important and actually significant data to appear and the other rare and sporadic events to be of less statistical value

The group with normal progress of labor acted as a base line to assess the normal values in the Egyptian population which was not assessed before.

\section{Weaknesses of the study}

dVEs were done by multiple birth attendants with variable degrees of experience and training. This may have influenced the results concerning the reproducibility and accuracy of the dVE when compared to the intrapartum ultrasound which was done by a single, more experienced operator

We calculated the whole duration of labor depending on the duration of $1^{\text {st }}$ and $2^{\text {nd }}$ stages.

Evaluation of the intrapartum ultrasound would have been more accurate if time to delivery was to be considered

By considering the successful outcome of labor to be spontaneous vaginal delivery, the fetal outcome was not taken into consideration and thus any adverse perinatal outcome (although very rarely occurring) may have impacted our results.

\section{Limitations of the study}

- $\quad$ Performance of the ultrasounds by a single operator, even if the latter situation was necessary to eliminate interobserver bias, limited the ability of the research team to involve more subjects in the study

\section{References}

1. Williams JW, Cunningham FG, Leveno KJ, Bloom SL, Spong CY, Dashe JS. Williams Obstetrics; 2018.

2. Neal JL, Lowe NK. Physiologic partograph to improve birth safety and outcomes among low-risk, nulliparous women with spontaneous labor onset. Med Hypotheses. 2012;78(2):319-26. http://doi.org/10.1016/j.mehy.2011.11.012

PMid:22138426

3. Hassan WA, Eggebø T, Ferguson M, Gillett A, Studd J, Pasupathy $D$, et al. The sonopartogram: A novel method for recording progress of labor by ultrasound. Ultrasound Obstet Gynecol. 2014;43(2):189-94. http://doi.org/10.1002/uog.13212 PMid:24105734

4. Hjartardóttir H, Lund SH, Benediktsdóttir S, Geirsson RT, Eggebø TM. Fetal descent in nulliparous women assessed by ultrasound: A longitudinal study. Am J Obstet Gynecol. 2021;224(4):378.e1-15.

PMid:33039395

5. Ghi T, Eggebø T, Lees C, Kalache K, Rozenberg P, Youssef A, et al. ISUOG Practice Guidelines: Intrapartum ultrasound. Ultrasound Obstet Gynecol. 2018;52(1):128-39.

PMid:29974596

6. Gizzo S, Andrisani A, Noventa M, Burul G, Di Gangi S, Anis $O$, et al. Intrapartum ultrasound assessment of fetal spine position. Biomed Res Int. 2014;2014:783598. https://doi. org/10.1155/2014/783598.

7. World Health Organization. Education material for teachers of midwifery. Midwifery Educ Modul. 2008;12:75.

8. Youssef A, Salsi G, Montaguti E, Bellussi F, Pacella G, Azzarone $\mathrm{C}$, et al. Automated measurement of the angle of progression in labor: A feasibility and reliability study. Fetal Diagn Ther. 2017;41(4):293-9. http://doi.org/10.1159/000448947 PMid:27592216

9. Eggebø TM, Hassan WA, Salvesen KA, Lindtjørn E, Lees CC. Sonographic prediction of vaginal delivery in prolonged labor: A two-center study. Ultrasound Obstet Gynecol. 2014;43(2):195201. http://doi.org/10.1002/uog.13210 PMid:24105705

10. Tutschek B, Torkildsen EA, Eggebø TM. Comparison between ultrasound parameters and clinical examination to assess fetal head station in labor. Ultrasound Obstet Gynecol. 2013;41(4):425-9. http://doi/org/10.1002/uog.12422

PMid:23371409 
11. Egypt Demographic and Health Survey 2014. Cairo, Egypt: Ministry of Health and Population and ICF International; 2015.

12. Wiafe YA, Whitehead B, Venables H, Nakua EK. The effectiveness of intrapartum ultrasonography in assessing cervical dilatation, head station and position: A systematic review and meta-analysis. Ultrasound. 2016;24(4):222-32. http://doi.org/10.1177/1742271X16673124

PMid:27847537 